\title{
Comparison of the Effect of Hot and Thermo-Neutral Environments on Fat Oxidation during Post-Exercise Recovery in Exercise-Trained Obese Women: A Preliminary Report
}

\author{
Parimon Kaewpaluk ${ }^{1}$, Onanong Kulaputana $a^{1,2,3, *}$ and \\ Sompol Sanguanrungsirikul ${ }^{1,2,3}$
}

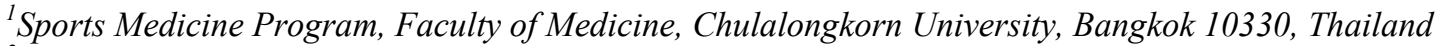 \\ ${ }^{2}$ Department of Physiology, Faculty of Medicine, Chulalongkorn University, Bangkok 10330, Thailand \\ ${ }^{3}$ King Chulalongkorn Memorial Hospital, Bangkok 10330, Thailand
}

\author{
('Corresponding author's e-mail: Onanong.K@chula.ac.th)
}

Received: 22 March 2020, Revised: 13 March 2021, Accepted: 22 March 2021

\begin{abstract}
Exercise training is recommended to promote energy expenditure. Fat utilization occurs during exercise and continues for an extended period of time after the exercise session. The environmental temperatures can influence whole body substrate oxidation. The present study aimed to address the impacts of environmental temperature on fat oxidation during post-exercise recovery in exercise-trained obese women. Eleven sedentary obese women (age: 18 - $50 \mathrm{y}$, BMI: $27.5-40 \mathrm{~kg} / \mathrm{m}^{2}$ ) with regular menstruation participated in the study. All subjects underwent a 4-week moderate-intensity aerobic exercise program. After training, each subject completed 2 occasions of post-exercise recovery testing in hot $\left(31-32{ }^{\circ} \mathrm{C}\right)$ and thermo-neutral $\left(22-23{ }^{\circ} \mathrm{C}\right)$ conditions in a randomized crossover fashion with $3-4$ days of washout period. Two exercise bouts preceding each recovery condition were identically performed for $60 \mathrm{~min}$ at $60 \%$ of heart rate reserve $\left(\mathrm{HR}_{\text {reserve }}\right)$ in the thermo-neutral condition. Both experiments were conducted during the follicular phase of menstrual cycle. Substrate oxidations were determined during $1 \mathrm{~h}$ of post-exercise recovery using indirect calorimetry. The results showed that the fat oxidation during recovery in thermo-neutral environment $\left(52.8 \pm 26.5 \mathrm{mg} \cdot \mathrm{kg}^{-1} \cdot \mathrm{h}^{-1}\right)$ was significantly greater than recovery in hot environment $\left(32.3 \pm 27.9 \mathrm{mg} \cdot \mathrm{kg}^{-1} \cdot \mathrm{h}^{-1}, p=0.0002\right)$. Total energy from substrate oxidation was not different between hot and thermo-neutral environments. Thus, in obese women with 4-week exercise training, recovery in the thermo-neutral condition has a higher fat oxidation than in the hot condition. This result may be implicated in weight management for temperature of choice to recover after routine exercise training sessions.
\end{abstract}

Keywords: Post-exercise recovery, Fat oxidation, Indirect calorimetry, Obesity, Moderate-intensity exercise training, Environmental temperature

\section{Introduction}

Obesity has become a global epidemic and a significant public health problem, leading to many chronic diseases such as cardiovascular disease, stroke, diabetes, and hypertension [1]. The World Health Organization (WHO) reported the worldwide death of these diseases to be about 2.8 million people per year in overweight and obese people [2]. The leading causes of obesity are imbalanced energy (positive energy balance) and having a sedentary lifestyle [3,4]. The American College of Sports Medicine (ACSM) recommended moderate-intensity aerobic exercise, for $30-60 \mathrm{~min} /$ day, more than or equal to 5 days/week accumulated to $150-300 \mathrm{~min} /$ week in overweight and obese people [5].

Exercise increases energy expenditure not only during exercises but also during recovery after the exercise session. The intensity and duration of exercise have been shown to influence fat oxidation portion of energy expenditure during post-exercise recovery in healthy women [6]. The longer duration of exercise had more effect on recovery fat oxidation than the shorter duration of exercise [6]. In addition, the recovery fat oxidation was increased at a greater extent in high intensity compared to low to moderate intensity exercise [7].

Research has shown that fat oxidation during rest and exercise is affected by environmental temperature [8-11]. Moreover, a recent study showed that environmental temperature can influence fat 
oxidation during post-exercise recovery in sedentary obese women [12]. Contribution of fat oxidation to the energy expended during recovery after a moderate-intensity exercise session was higher in thermoneutral environment $\left(24-25^{\circ} \mathrm{C}\right)$ than in hot temperature $\left(31-32{ }^{\circ} \mathrm{C}\right)$ [12]. This information may be an implication for weight management with exercise in obese women.

Exercise training has been shown to have an affect towards fat oxidation. Moderate-intensity exercise training between 2 - 10 weeks increased rate of fat oxidation during exercise in obese men and overweight women [13-15]. Additionally, exercise training for $6-8$ weeks also increased fat oxidation during rest time in overweight and obese subjects $[16,17]$. Taken together, exercise training and the temperature can affect fat oxidation at rest and during exercise. Since environmental temperature affected fat oxidation during post-exercise recovery in untrained obese women, it is unclear whether fat oxidation during post-exercise recovery differently affected by environmental temperatures in exercise-trained obese women. This is important particularly to the study in the obese subjects who live in hot and humid countries such as Thailand. In addition, the information in obese women is limited despite the fact that the high prevalence of obesity occurs in females [18-21], yet most research studies were in males. Therefore, the present study aimed to investigate the effect of environmental temperatures on fat oxidation during post-exercise recovery in obese women who had participated in an aerobic exercise training program. Hence, the specific research question was asked. Does fat oxidation during post-exercise recovery differ between thermo-neutral and hot environmental conditions in obese women after a short-term (4 weeks) exercise training? The results would likely be useful for obese women to enhance fat utilization and body weight management with exercise training.

\section{Materials and methods}

\section{Research method}

The present study involved an experimental research design in which a comparison of fat oxidation during post-exercise recovery between 2 environmental conditions was determined in obese women after an aerobic exercise training. The participants who passed all eligibility criteria underwent an exercise training program for 4 weeks. After training, they performed 2 post-exercise training tests in different occasions. Each test occasion included an acute bout of moderate-intensity exercise at $60 \%$ of heart rate reserve $\left(\mathrm{HR}_{\text {reserve }}\right)$ for $60 \mathrm{~min}$ followed by a 60 -min recovery phase. The recovery phase was performed in 2 environmental temperatures with randomized crossover sequence (thermo-neutral and then hot temperatures or vice versa). Additionally, the participants would wear a mobile gas analyzer module for expired gas analyses throughout the experiments. The study protocol was approved by the Institutional Review Board, the Faculty of Medicine, Chulalongkorn University (IRB No.698/61). All subjects provided informed consent prior to participating the study.

\section{Participants}

All participants were recruited from the research center surrounding areas by posters and flyers. Eleven obese women (BMI $27.5-40 \mathrm{~kg} / \mathrm{m}^{2}$ ), aged 18 - 50 years old, who had no high blood pressure, diabetes, cardiac disease, or dyslipidemia were recruited for the study. The subjects had to have regular menstrual cycles in the past 3 months [22]. The use of contraceptive hormones or any diet pills was excluded. Furthermore, the subjects were eligible if they did not have the body weight changes of more than $3 \mathrm{~kg}$ in the past 6 months [23,24].

This study was a preliminary report of a larger study designed to determine the effect of exercise training on substrate oxidation in obese subjects. The study protocol was approved by the Institutional Review Board, the Faculty of Medicine, Chulalongkorn University (IRB No.698/61). All subjects provided informed consent prior to participating the study.

\section{Diet stabilization}

The subjects underwent the diet stabilization for a period of 4 weeks prior to participating in the moderate-intensity exercise training program. This would minimize the influence of dietary variation on the study results. Diet stabilization involved a healthy diet education by a nutritionist. After that, the subjects provided 3 days of diet record each week. The food log was handed to the nutritionist every week for 4 weeks for nutritional evaluation and the feedback of their food calories and nutrition was given to the subjects individually. This diet practice was maintained throughout the exercise training period. 


\section{Exercise training program}

The exercise training program involved a moderate-intensity walking exercise on a treadmill. The intensity and duration started from $50 \% \mathrm{HR}_{\text {reserve }}$ for $30 \mathrm{~min}$ and progressed to $60 \% \mathrm{HR}_{\text {reserve }}$ for $60 \mathrm{~min}$. The participants would exercise 4 sessions each week in the ambient temperature of $22-23{ }^{\circ} \mathrm{C}$. All training sessions were supervised. All subjects completed the 4-week training program (Figure 1).

\begin{tabular}{|c|c|c|c|c|c|c|c|c|c|c|c|c|c|c|c|c|c|c|c|c|c|c|c|c|}
\hline & \multicolumn{7}{|c|}{ Week 1} & \multicolumn{6}{|c|}{ Week 2} & \multicolumn{6}{|c|}{ Week 3} & \multicolumn{5}{|c|}{ Week 4} \\
\hline & 1 & 2 & 3 & 4 & 5 & 6 & 7 & 8 & 9 & 10 & 11 & 12 & $13 \quad 14$ & 1 & 516 & 17 & 18 & 19 & $20 \quad 21$ & 22 & 23 & $\begin{array}{l}24 \quad 25 \\
24\end{array}$ & 262 & $27 \quad 28$ \\
\hline Exercige session (S) & & S1 & S2 & & S3 & S4 & & & S5 & $S B$ & & S7 & SB & & s: & 510 & & S11 s & $\mathrm{S} 12$ & & & S14 & & S16 \\
\hline Intensity (\%HRR) & & 50 & 50 & & 50 & 50 & & & 55 & 55 & & 55 & 55 & & $a$ & 60 & & 60 & & & 60 & 60 & 606 & 60 \\
\hline Time (min) & & 30 & 30 & & 35 & 40 & & & 40 & 45 & & 50 & 50 & & 5 & 55 & & 60 & 60 & & 60 & 60 & 608 & 60 \\
\hline
\end{tabular}

Figure 1 Progressive moderate-intensity exercise training program.

\section{Substrate oxidation}

After the exercise training program, substrate (fat and carbohydrate) oxidations were determined in in the morning of 2different occasions. The tests were performed in the follicular phase $\left(1^{\text {st }}-7^{\text {th }}\right.$ days) of the menstruation period to control for the potential effect of ovarian hormones on substrate metabolism. The substrate oxidations were tested during $1 \mathrm{~h}$ of post-exercise recovery period on 2 different ambient temperatures (thermo-neutral or hot temperatures) in a randomized crossover fashion. Each session of the post-exercise recoveries followed a 60 -min moderate-intensity exercise $\left(60 \% \mathrm{HR}_{\text {reserve }}\right)$ in thermo-neutral temperature. The substrate oxidation rates were determined using a portable gas analyzer (Jaeger, Oxycon mobile, Germany) and calculated by Peronnet and Massicotte formula [25]. For the period of $24 \mathrm{~h}$ prior to each testing day all subjects consumed the same food items with identical amount of calories provided by the investigators.

\section{Statistical analysis}

The data were analyzed using the statistical package for the social science (SPSS), version 22.0 for windows. All data are reported as mean $\pm \mathrm{SD}$. The linear mixed model for a crossover trial was used to test the differences between the substrate oxidations during recovery in hot and in thermo-neutral environments. Statistical significance was accepted for all tests at $p<0.05$.

\section{Results and discussion}

A total of 11 subjects completed the study. The characteristics of 11 subjects are shown in Table1.

Table 1 Characteristics of 11 subjects (Mean \pm SD).

\begin{tabular}{ccc}
\hline Characteristics & Mean \pm SD & Range \\
\hline Age $(\mathrm{y})$ & $33.9 \pm 7.0$ & $23-43$ \\
Weight $(\mathrm{kg})$ & $86.4 \pm 13.0$ & $71.4-107.4$ \\
Height $(\mathrm{m})$ & $1.6 \pm 0.1$ & $1.5-1.6$ \\
BMI $\left(\mathrm{kg} / \mathrm{m}^{2}\right)$ & $33.8 \pm 3.7$ & $29.7-39.7$ \\
Body fat $(\%)$ & $46.0 \pm 3.8$ & $40.2-51.3$ \\
Fasting blood glucose (mg/dL) & $91.3 \pm 5.4$ & $82-98$ \\
HDL-C (mg/dL) & $47.7 \pm 6.0$ & $40-60$ \\
LDL-C (mg/dL) & $115.9 \pm 12.7$ & $89-131.6$ \\
Triglycerides (mg/dL) & $103.9 \pm 24.9$ & $48-139$ \\
\hline BMI
\end{tabular}

BMI $=$ Body mass index, HDL-C $=$ High density lipoprotein cholesterol, LDL-C $=$ Low density lipoprotein cholesterol 
The average volume of training was $12.8 \pm 1.2$ sessions. The mean temperature and humidity during the exercise were $22.7 \pm 0.4{ }^{\circ} \mathrm{C}$ and $69.2 \pm 3.1 \%$. The mean temperature and humidity during recovery in thermo-neutral and hot conditions are shown in Table 2.

Table 2 The environmental temperature and relative humidity during recovery.

\begin{tabular}{ccc}
\hline Environment during recovery & Temperature $\left({ }^{\circ} \mathbf{C}\right)$ & Relative humidity $(\%)$ \\
\hline Thermo-neutral & $22.6 \pm 0.1$ & $71.3 \pm 1.7$ \\
Hot & $31.6 \pm 0.4$ & $68.4 \pm 2.7$ \\
\hline
\end{tabular}

Substrate oxidations during post post-exercise recovery in both environments were demonstrated in Table 3. The fat oxidation during $1 \mathrm{~h}$ recovery in a thermo-neutral environment was significantly higher than in a hot environment $(p=0.0002)$. The carbohydrate oxidation during recovery was lower in thermoneutral than in hot environments $(p=0.002)$. The difference of energy expenditure during the recovery period in both environments was not statistically significant $(p>0.05)$.

Table 3 Substrate oxidations and energy expenditure during recovery in thermo-neutral and hot environments $(\mathrm{n}=11)$.

\begin{tabular}{cccc}
\hline & Thermo-neutral environment & Hot environment & $p$-value \\
\hline $\begin{array}{c}\text { Recovery Fat oxidation } \\
\left(\mathrm{mg} \cdot \mathrm{kg}^{-1} \cdot \mathrm{h}^{-1}\right)\end{array}$ & $52.8 \pm 26.5$ & $32.3 \pm 27.9$ & 0.0002 \\
$\begin{array}{c}\text { Recovery CHO oxidation } \\
\left(\mathrm{mg} \cdot \mathrm{kg}^{-1} \cdot \mathrm{h}^{-1}\right)\end{array}$ & $71.8 \pm 41.7$ & $108.5 \pm 40.6$ & 0.002 \\
$\begin{array}{c}\text { Energy Expenditure } \\
\left(\mathrm{kcal} \cdot \mathrm{h}^{-1}\right)\end{array}$ & $64.2 \pm 11.8$ & $60.4 \pm 15.1$ & 0.438 \\
\hline
\end{tabular}

Values as mean \pm standard deviation.

A

Total energy expenditure Thermo-neutral environment

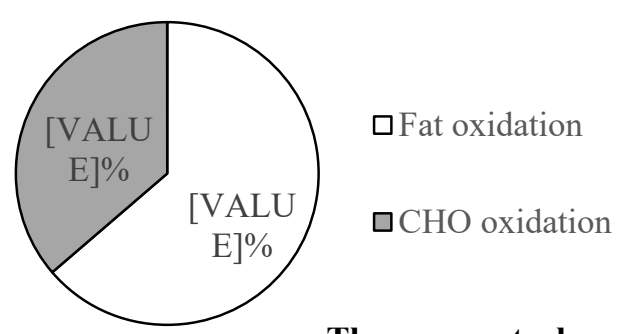

Thermo-neutral
B Total energy expenditure Hot environment

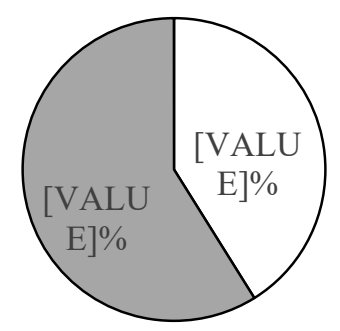

$\square$ Fat oxidation

$\square \mathrm{CHO}$ oxidation

Hot

Figure 2 Total energy expenditure in A) thermo-neutral environment and B) hot environment.

The present study showed an important finding that in obese women, the recovery fat oxidation after $60 \mathrm{~min}$ of moderate-intensity $\left(60 \% \mathrm{HR}_{\text {reserve }}\right)$ exercise was greater in the thermo-neutral environment than in the hot environment. This was similar to the result of the previous study on recovery fat oxidation after a single bout of moderate-intensity exercise $\left(45-50 \% \mathrm{HR}_{\text {reserve }}, 30 \mathrm{~min}\right)$ in untrained obese women 
[12]. The result of the present study added a significant piece of knowledge to the previous study as it demonstrated that the greater fat oxidation during recovery in thermo-neutral condition continued to occur after a period of 4-week training. Thus, the implication is that obese people appear to oxidize more fat during their recovery period after each exercise bout by doing the recovery in the thermo-neutral environment. This benefit likely sustains throughout the course of exercise training for at least 4 weeks.

It has been suggested that fat oxidation which occurred during the recovery after finishing exercise was related to glycogen re-synthesis in the muscle [26-29]. In hot environment, glycogen re-synthesis during the recovery period was impaired due to low glucose transport [30]. Furthermore, Pi-mediated activation of glycogen phosphorylase led to inhibition of the glycogen accumulation in hot temperature $[30,31]$. Thus, the glycogen re-synthesis in the hot environment appeared to be less effectively restored $[30,31]$. In the present study, we reported the influence of temperature on fat oxidation during the recovery phase in exercise-trained obese women. The recovery fat oxidation in cold temperature tended to increase after the exhaustive exercise [11]. Glycogen re-synthesis, which uses the free fatty acids from fat oxidation to be the substrate in the process, possibly occurs to a greater extent during recovery in the cold environment than in the hot environment.

In the recovery phase, it is well accepted that there is an excess post-exercise oxygen consumption (EPOC). This causes enhanced energy expenditure above resting level compatible with the findings in the present study. The oxygen consumption increased in the first $2 \mathrm{~h}$ since more oxygen was brought into the body to clear the lactic acid from the bloodstream, to recover the oxygen reserve, and to restore the ATP/creatine phosphate, thermoregulation and homeostasis [32-35]. In the present study, the total energy expenditure during recovery in the thermo-neutral environment tended to increase more than recovery in the hot environment; however, the difference was not significantly different. It is probable that the temperatures employed in the present study were not too extreme and the duration was not too extensive. When the human body exposed to the cold temperature for an extended period of time, triglycerides are broken down into free fatty acids and glycerol to provide energy for shivering [36-39]. The environment in the present study was similar to the fitness center $\left(22-23{ }^{\circ} \mathrm{C}\right)$, which was the temperature generally exposed by people within the office building or department store; therefore, the total energy expenditure was obviously unchanged.

The novel finding in obese women in the present study was that fat oxidation was greater during recovery in the thermo-neutral environment. It should be noted that exercise trained subjects are likely to increase more recovery fat oxidation after exercising than untrained individuals [40]. In addition, highly trained individuals showed more fat oxidation than moderately trained individuals [41]. The present study demonstrated that enhanced fat oxidation continued in the post-exercise period in the obese women who engaged in exercise training for 4 weeks. This suggests that the recovery in a thermo-neutral environment would yield more benefits to the obese population who employ exercise training as their weight management program. It is possible that when obese individuals progressively continue their training program, fat oxidation during post-exercise recovery would be further increased. However, this hypothesis is left to be proven in future research.

Limitation should be pointed out as follows. The present study employs a moderate-intensity exercise for the training program. It is not known whether other exercise training programs would yield a similar result. Moreover, when the exercise training program is maintained for a more extended period, it is unknown that fat oxidation would still be elevated with recovery in the thermoneutral environment.

\section{Conclusions}

The recovery fat oxidation after 4-week progressive moderate-intensity exercise training was higher in the thermo-neutral environment than in the hot environment. This finding was likely beneficial for obese women, whose habitats are in hot and humid countries, to select the appropriate environment to enhance fat oxidation with exercise.

\section{Acknowledgments}

This study was supported by Ratchadaphiseksomphot Endowment Fund (RA62/028) from the Faculty of Medicine, Chulalongkorn University, and the CU. Graduate school thesis grant (GCUGR1225623010M No.010) from Chulalongkorn University, Thailand. 


\section{References}

[1] World Health Organization. Appropriate body-mass index for Asian populations and its implications for policy and intervention strategies. Lancet 2004; 363, 157-63.

[2] World Health Organization. Global status report on noncommunicable diseases 2010. World Health Organization, Geneva, Switzerland, 2011.

[3] E Levadoux, B Morio, C Montaurier, V Puissant, Y Boirie, N Fellmann, B Picard, P Rousset, B Beaufrere and P Ritz. Reduced whole-body fat oxidation in women and in the elderly. Int. J. Obes. 2001; 25, 39-44.

[4] B Purkart, B Šimunič and M Gerževič. An overview of training methods that promote the highest lipid oxidation during and after a single exercise session. Ann. Kinesiol. 2016; 6, 93-118.

[5] American College of Sports Medicine. ACSM's guidelines for exercise testing and prescription. $10^{\text {th }}$ ed. Wolters Kluwer, Philadelphia, 2018, p. 287-90.

[6] A Warren, EJ Howden, AD Williams, JW Fell and NA Johnson. Postexercise fat oxidation: Effect of exercise duration, intensity, and modality. Int. J. Sport Nutr. Exerc. Metabol. 2009; 19, 607-23.

[7] JF Phelain, E Reinke, MA Harris and CL Melby. Postexercise energy expenditure and substrate oxidation in young women resulting from exercise bouts of different intensity. J. Am. Coll. Nutr. 1997; 16, 140-6.

[8] J Katoh, Y Hara, M Kurusu, J Miyaji, K Ishihara, A Tutoh, T Kazumi and H Taniguchi. Respiratory effects on exercise and whole thermotolerance (sauna) stress in obese patients. J. Phys. Ther. Sci. $1996 ; 8,25-7$.

[9] P Tikuisis, I Jacobs, D Moroz, AL Vallerand and L Martineau. Comparison of thermoregulatory responses between men and women immersed in cold water. J. Appl. Physiol. 2000; 89, 1403-11.

[10] F Haman, F Peronnet, GP Kenny, D Massicotte, C Lavoie, C Scott and JM Weber. Effect of cold exposure on fuel utilization in humans: Plasma glucose, muscle glycogen, and lipids. J. Appl. Physiol. 2002; 93, 77-84.

[11] AS Weller, PL Greenhaff and LA Macdonald. Physiological responses to moderate cold stress in man and the influence of prior prolonged exhaustive exercise. Exp. Physiol. 1998; 83, 679-95.

[12] O Kulaputana, T Soythong, S Sanguanrangsirikul and T Sooksirivoraboot. Environmental temperature affects fat and carbohydrate oxidations during recovery period after moderate intensity exercise in obese women. J. Med. Assoc. Thai. 2020; 103, 240-7.

[13] MC Venables and AE Jeukendrup. Endurance training and obesity: Effect on substrate metabolism and insulin sensitivity. Med. Sci. Sports Exerc. 2008; 40, 495-502.

[14] S Lanzi, F Codecasa, M Cornacchia, S Maestrini, P Capodaglio, A Brunani, P Fanari, A Salvadori and D Malatesta. Short-term HIIT and fat max training increase aerobic and metabolic fitness in men with class II and III obesity. Obesity (Silver Spring) 2015; 23, 1987-94.

[15] S Tan, J Wang, L Cao, Z Guo and Y Wang. Positive effect of exercise training at maximal fat oxidation intensity on body composition and lipid metabolism in overweight middle-aged women. Clin. Physiol. Funct. Imag. 2016; 36, 225-30.

[16] E Lefai, S Blanc, I Momken, E Antoun, I Chery, A Zahariev, L Gabert, A Bergouignan and C Simon. Exercise training improves fat metabolism independent of total energy expenditure in sedentary overweight men, but does not restore lean metabolic phenotype. Int. J. Obes. 2017; 41, 1728-36.

[17] G Jabbour, P Mauriege, D Joanisse and HD Iancu. Effect of supramaximal exercise training on metabolic outcomes in obese adults. J. Sports Sci. 2017; 35, 1975-81.

[18] M Ng, T Fleming, M Robinson, B Thomson, N Graetz, C Margono, et al. Global, regional, and national prevalence of overweight and obesity in children and adults during 1980 - 2013: A systematic analysis for the global burden of disease study 2013 . Lancet $2014 ; \mathbf{3 8 4}, 766-81$.

[19] World Health Organization. Global status report on noncommunicable diseases 2014. World Health Organization, Geneva, Switzerland, 2014.

[20] H Yatsuya, Y Li, EH Hilawe, A Ota, C Wang, C Chiang, Y Zhang, M Uemura, A Osako, Y Ozaki, and A Aoyama. Global trend in overweight and obesity and its association with cardiovascular disease incidence. Circ. J. 2014; 78, 2807-18.

[21] W Aekplakorn and L Mo-Suwan. Prevalence of obesity in Thailand. Obes. Rev. 2009;10, 589-92.

[22] N Santoro, B Lasley, D McConnell, J Allsworth, S Crawford, EB Gold, JS Finkelstein, GA Greendale, J Kelsey, S Korenman, JL Luborsky, K Matthews, R Midgley, L Powell, J Sabatine, M Schocken, MF Sowers and G Weiss. Body size and ethnicity are associated with menstrual cycle 
alterations in women in the early menopausal transition: The Study of Women's Health across the Nation (SWAN) daily hormone study. J. Clin. Endocrinol. Metab. 2004; 89, 2622-31.

[23] JL Keller, PR Casson and MJ Toth. Relationship of androgens to body composition, energy and substrate metabolism and aerobic capacity in healthy, young women. Steroids 2011; 76, 1247-51.

[24] TDS Ferreira, JFR Fernandes, LDS Araújo, LP Nogueira, PM Leal, VP Antunes, MDLG Rodrigues, DCT Valença, SE Kaiser and MRST Klein. Serum uric acid levels are associated with cardiometabolic risk factors in healthy young and middle-aged adults. Arq. Bras. Cardiol. 2018; 111, 833-40.

[25] F Peronnet and D Massicotte. Table of nonprotein respiratory quotient: An update. Can. J. Sport Sci. 1991; 16, 23-9.

[26] NE Kimber, GJF Heigenhauser, LL Spriet and DJ Dyck. Skeletal muscle fat and carbohydrate metabolism during recovery from glycogen-depleting exercise in humans. J. Physiol. 2003; 548, 919-27.

[27] B Egan and JR Zierath. Exercise metabolism and the molecular regulation of skeletal muscle adaptation. Cell Metab. 2013; 17, 162-84.

[28] JN Nielsen and EA Richter. Regulation of glycogen synthase in skeletal muscle during exercise. Acta Physiol. Scand. 2003; 178, 309-19.

[29] B Kiens and EA Richter. Utilization of skeletal muscle triacylglycerol during postexercise recovery in humans. Am. J. Physiol. 1998; 275, E332-E337.

[30] SJ Blackwood, E Hanya and A Katz. Effect of postexercise elevation on postexercise glycogen metabolism of isolated mouse soleus muscle. J. Appl. Physiol. 2019; 126, 1103-09.

[31] M Naperalsky, B Ruby and D Slivka. Environmental temperature and glycogen resynthesis. Int. J. Sports Med. 2010; 31, 561-6.

[32] JR Speakman and C Selman. Physical activity and resting metabolic rate. Proc. Nutr. Soc. 2003; 62, 621-34.

[33] GA Gaesser and GA Brooks. Metabolic bases of excess post-exercise oxygen consumption: A review. Med. Sci. Sports Exerc. 1984;16, 29-43.

[34] TJ Horton, MJ Pagliassotti, K Hobbs and JO Hill. Fuel metabolism in men and women during and after long-duration exercise. J. Appl. Physiol. 1998; 85, 1823-32.

[35] E Borsheim and R Bahr. Effect of exercise intensity, duration and mode on post-exercise oxygen consumption. Sports Med. 2003; 33, 1037-60.

[36] J Himms-Hagen. Sympathetic regulation of metabolism. Pharmacol. Rev. 1967; 19, 367-461.

[37] AJ Young, SR Muza, MN Sawka, RR Gonzalez and KB Pandolf. Human thermoregulatory responses to cold air are altered by repeated cold water immersion. J. Appl. Physiol. 1986; 60, 15428.

[38] AL Vallerand and I Jacobs. Rates of energy substrates utilization during human cold exposure. Eur. J. Appl. Physiol. Occup. Physiol. 1989; 58, 873-8.

[39] AL Vallerand and I Jacobs. Influence of cold exposure on plasma triglyceride clearance in humans. Metabolism 1990; 39, 1211-8.

[40] AB Stisen, O Stougaard, J Langfort, JW Helge, K Sahlin and K Madsen. Maximal fat oxidation rates in endurance trained and untrained women. Eur. J. Appl. Physiol. 2006; 98, 497-506.

[41] J Achten and AE Jeukendrup. Maximal fat oxidation during exercise in trained men. Int. J. Sports Med. 2003; 24, 603-8. 